\title{
A Dynamical Mechanism for the Evolution and Breakdown of Cooperation in the Snowdrift Game in Adaptive Networks
}

\author{
Gerd Zschaler $^{1}$, Arne Traulsen ${ }^{2}$, and Thilo Gross ${ }^{1}$ \\ 1 Max-Planck-Institut für Physik komplexer Systeme, \\ Nöthnitzer Str. 38, 01187 Dresden, Germany \\ zschalerapks.mpg. de \\ 2 Max-Planck-Institut für Evolutionsbiologie, \\ August-Thienemann-Str. 2, 24306 Plön, Germany
}

\begin{abstract}
We present a novel dynamical mechanism promoting cooperation in the snowdrift game on an adaptive network. In infinite systems, asymptotic full cooperation can be achieved, while sudden breakdowns of highly cooperative states are observed in finite systems.
\end{abstract}

We consider the evolutionary dynamics of the snowdrift game on an adaptive network, where the coupling between strategy evolution and topological evolution provides a mechanism promoting cooperation dynamically and leading to asymptotic full cooperation in the limit of infinite system size.

In our model, the nodes of an undirected network represent agents and the links represent interactions among them. Each agent is assigned a strategy, either unconditional cooperation or unconditional defection. The agents' interactions are described in terms of a pairwise cooperative game, namely the snowdrift game. In this game, an agent receives an abstract payoff that is determined by the pairing of its own and the opponent's strategy. Each agent obtains a total payoff from all the interactions with its neighbors. While mutual cooperation in each interaction is the social optimum, unilateral defection yields a higher payoff and undermines the evolution of cooperation. For this reason, additional mechanisms are typically required to allow cooperators to thrive in evolutionary games [1-3].

The evolution of the system is driven by two processes: the agents can imitate the strategies of successful neighbors or cut the link to a less successful neighbor and reconnect it to a random agent. In contrast to previous work that mainly focused on local update rules [4-6], the agents use non-local information about the general performance of the strategies to assess the success of a strategy in our model. The agents thus update their strategies by imitating what they perceive as the more successful one within their "information horizon", i.e., within a neighborhood of a fixed radius. In the same way, they rewire their links depending on this non-local information. Both processes are associated with rate constants, which define their relative time scales, and selection intensities, which control how strongly they are influenced by the performance of the agents' strategies. Thus, the strategy dynamics feed back on the topological evolution of the system, rendering it an adaptive network [7]. 
The adaptive nature of the model leads to a novel dynamical mechanism promoting cooperation. In full simulations of the network, we find oscillations in the number of cooperating players when the link rewiring rate is greater than a critical value. With increasing rewiring rate, or information horizon, the amplitude and period of these oscillations also increase. In the limit of infinite system size, the time-averaged payoff of the cooperators equals that of the defectors in this regime. Nevertheless, for the case of infinite information horizon, a state of full cooperation is approached asymptotically if the rate of topological change exceeds a finite threshold. Using a moment expansion approach and pair approximation [8, 9], we derive a low-dimensional analytical model relating this phenomenon to the bifurcation structure of the underlying dynamical system. Asymptotic full cooperation is thus achieved by the formation of a homoclinic loop in a global bifurcation, which is triggered by sufficiently fast and strongly selective rewiring. Furthermore we show that in finite populations, this mechanism can lead to periods of almost full cooperation interrupted by recurrent collapses to episodes of predominant defection.

A sufficiently large information horizon is necessary to observe the homoclinic transition in our model. We note, however, that this assumption is not necessary for the oscillations to appear, even though it is conceivable that global information can be accessible in social systems through, e.g., general beliefs, rumors, or the mass media. If the agents' information access is restricted to small finite information horizons, the present model still exhibits oscillations. Furthermore, a transition similar to the homoclinic bifurcation was found numerically in a different model [10], in which local update rules are assumed. In our model, asymptotic full cooperation is achieved dynamically by the interplay between topological and strategical evolution, rather than by assembling characteristic "cooperator-friendly" topologies. In finite populations, fluctuations destabilize highly cooperative states, leading to spontaneous collapses of the latter. The homoclinic mechanism in combination with noise may thus be a relevant ingredient in the systemic failure of cooperation that is found in real-world systems [11].

\section{References}

[1] Nowak, M.A., Sigmund, K.: Science 303, 793-799 (2004)

[2] Doebeli, M., Hauert, C.: Ecol. Lett. 8, 748-766 (2005)

[3] Macy, M.W., Flache, A.: Proc. Natl. Acad. Sci. U. S. A. 99, 7229-7236 (2002)

[4] Zimmermann, M.G., Eguíluz, V.M., San Miguel, M., Spadaro, A.: Adv. Complex Syst. 3, $283(2000)$

[5] Santos, F.C., Pacheco, J.M., Lenaerts, T.: Proc. Natl. Acad. Sci. U. S. A. 103, 3490-3494 (2006)

[6] Pacheco, J.M., Traulsen, A., Nowak, M.A.: Phys. Rev. Lett. 97, 258103 (2006)

[7] Gross, T., Blasius, B.: J. R. Soc. Interface 5, 259-271 (2008)

[8] Gross, T., D’Lima, C.J.D., Blasius, B.: Phys. Rev. Lett. 96, 208701 (2006)

[9] Keeling, M.J., Eames, K.T.D.: J. R. Soc. Interface 2, 295 (2005)

[10] Szolnoki, A., Perc, M.: New J. Phys. 11, 093033 (2009)

[11] Turchin, P.: Historical Dynamics: Why States Rise and Fall. Princeton Studies in Complexity. Princeton University Press (2003) 